\title{
Personalisierte Medizin und ihre Triebkräfte
}

\author{
Peter Möhr-Buxtorf \\ Dr. med., Facharzt für Allgemeine Innere Medizin, Mitglied FMH
}

Personalisierte Medizin (PM) ist keine grundsätzlich neue Strategie, um Krankheiten vorzubeugen, sie zu diagnostizieren und zu behandeln. Das verfügbare Wissen, bekannte Heilmittel und Techniken wurden seit jeher eingesetzt, um für den individuellen $\mathrm{Pa}$ tienten das bestmögliche Resultat zu erreichen ( $M E$ Medicine). Konkrete Erfolge erzielte bis anfangs des 20. Jahrhunderts aber weniger die ärztliche Patientenbetreuung, sondern kollektive Massnahmen wie administrative Seuchenkontrolle, Impfkampagnen, Hebung des Lebensstandards, Verbesserung von Ernährung, Wohnen und öffentlicher Hygiene (WE Medicine) [1]*. Im letzten Jahrhundert erweiterten naturwissenschaftliche und empirisch kontrollierte Erkenntnisse unsere diagnostischen und therapeutischen Möglichkeiten enorm. Seit der Jahrtausendwende sind gentechnologische Innovationen dazugekommen. Dank dem Human Genome Project haben sich neue Erkenntnisse über genetische und nicht genetische Einflussfaktoren bei Gesundheit und Krankheit und eine bessere Einschätzung der Rolle der Epigenetik für Genotyp und Phänotyp ergeben. Sie weisen auf eine grosse Diversität hin - sind wir doch alle Mutanten [2]. Sie sollen nun in die individuelle Diagnostik und in einen Therapieplan eingebaut werden (Precision Medicine, «Medizin nach Mass»). Bezüglich Umsetzung stehen wir erst am Anfang.

Das zukünftige Potential der PM schätzen die Triebkräfte dahinter, nämlich Big Pharma, Big Data, Big Research und Big Biotechnology als immens ein.

La médecine personnalisée et ses moteurs

Nos principaux partenaires dans le domaine médical doivent impérativement restaurer la confiance qu'ils ont eux-mêmes contribué à miner par le passé. L'industrie pharmaceutique est la première concernée et fait d'ailleurs l'objet de cet article; suivent le big data et les biotechnologies. D'un point de vue général, la tâche des firmes pharmaceutiques consiste à proposer un éventail de médicaments dûment testés, utiles et bien tolérés à des prix acceptables. Cette recommandation est également valable pour la médecine personnalisée, qui continuera à être développée sous la houlette de I'EPFZ et des hautes écoles partenaires. Au moment d'orienter la recherche basée sur les technologies et leur développement, deux objectifs opposés sont souvent à l'œuvre, à savoir une maximisation du profit du côté de l'industrie d'une part, et le bien-être et les perspectives de vie des patients selon les personnes qui en prennent soin, de l'autre.

Ein Blick auf die Zielsetzungen von gewichtigen Akteuren ist aufschlussreich.

\section{Big Pharma, Geschäftsmodell}

Der globale Pharmamarkt ist seit Beginn der 80er Jahre stetig gewachsen und hat bis heute einen Umfang von über 500 Mia. USD/Jahr erreicht, wobei die Hälfte auf die USA entfällt. Daran partizipieren insbesondere die Top Fifteen (Big Pharma), unter denen Novartis Platz 1, Pfizer Platz 2 und Roche Platz 3 einnehmen [3]. Das Big Pharma-Geschäftsmodell (=Blockbuster-Modell) folgt seit der Reagan-Ära einer konsequenten Gewinnmaximierung innerhalb und ausserhalb der legalen Leitplanken. Margen von $15-20 \%$ sind üblich. Der Pharma-Gewinn überstieg noch 2003 den kumulierten Gewinn aller von Forbes gelisteten Industrien (Margen im Schnitt $~ 4 \%$ ). Blockbuster sind Medikamente mit Jahresumsätzen von 1 Mia. USD und mehr. Ein bis drei solcher Blockbuster auf dem Markt bilden die Geschäftsbasis einer Firma; weitere, in der Pipeline gestaffelte sichern den langfristigen Erfolg. Trotz angeblich enormen Forschungs- und Entwicklungskosten war die firmeneigene Innovationskraft noch nie brillant. Sie hält seit den 90er Jahren mit den Erwartungen überhaupt nicht mehr Schritt. Lizenzverträge mit auswärtigen Forschungsstellen, Firmenzukäufe und eigenmächtige Marktausweitungen für bereits etablierte Medikamente waren und sind die Folge [4, 5].

Grosse Pharma-Firmen suchen deswegen die Nähe zu staatlichen, mit Steuergeldern alimentierten Hochschulen (z.B. MIT, ETHZ) und deren Grundlagenforschern oder integrieren innovative Start-up-Firmen mit pfannenfertigen Produkten. Das spart ihnen Kosten in der präklinischen Phase der Medikamentenentwicklung [6]. Bezüglich anvisierten Krankheitsspektrums hat sich die Pharmakotherapie von chronischen Leiden und neu als Krankheiten definierten physiologischen $\mathrm{Zu}$ ständen als lukrativ erwiesen. Dies wird in der vollständigen Version dieses Artikels näher ausgeführt.* Besonders erfolgversprechend als Novitäten, da Forschungskosten einsparend und relativ risikoarm, sind Nachahmerprodukte eines bereits eingeführten Blockbusters ("Me-too drugs»). Sie machten bisher das Hauptgeschäft aus [1, 4]. 
Big Pharma schaltet sich in der klinischen Erprobungsphase der Medikamenten-Evaluation, besonders in Phase III, ein und wünscht, über alle Daten zu verfügen. Das erlaubt den Firmen, negative Studien nicht zu publizieren und bevorzugt positive für das Zulassungsprozedere und das Marketing zu verwenden. Falls sich ein verzerrtes Bild ergibt, werden die Biases im Geschäftsinteresse interpretiert. Übrigens sind rund die Hälfte aller Studien gar nie publiziert worden.

Der Patentschutz wird zäh verteidigt, seine Dauer ist beschränkt dehnbar. Er schiebt das Generikum-Stadium hinaus und verschafft dem neuen Arzneimittel eine lange Monopolstellung auf dem «freien» Markt [9]. Er vermag die Entwicklung von allenfalls billigeren Alternativpräparaten zu blockieren, z.B. durch Genpatentierung (HER-2 durch Genentech). Eines von fünf interessanten Genen geniesst ja bereits diesen privaten Patentschutz. Im Stadium der Zulassung wird seitens der Firmen viel Hintergrundarbeit, insbesondere informelle geleistet. Es geht um die Festlegung des Indikationsbereichs eines neuen, verschreibungspflichtigen Mittels, die vorschriftsgemässe Information von Fachwelt und Konsumenten, die Preisgestaltung und den Entscheid bezüglich Spezialitätenliste mit Kassenvergütung.

Nach einer Markteinführung schliesst die obligate Postmarketing-Phase (IV) an. Sie wird von den Firmen nicht bloss hinsichtlich Bewährung des Mittels im Praxisalltag und nicht bekannter oder bereits bekannter Nebenwirkungen, sondern vor allem fürs Marketing bei medizinischen Fachpersonen und beim Laien-Publikum intensiv genutzt.

Wir Ärzte - als «Gatekeeper» im System - werden bereits in der Ausbildungsphase, später in der Praxis von einem Heer persönlicher Pharmaberater informiert, umworben und mit Musterpackungen bedient. An den von der Industrie gesponserten Ärzte-Fortbildungsveranstaltungen - wo verlangte Weiterbildungspunkte erworben werden können - treten prominente, oft an den Studien beteiligt gewesene Forscher und Kliniker als Meinungsbildner auf. Ihre Instrumentalisierung ist teilweise bekannt [10, 11]. Dieselben Experten sind häufig an der Ausarbeitung von sog. Guidelines mitbeteiligt, welche die neuen Markenpräparate in die Praxis einordnen sollen. Beachtung oder Nichtbeachtung solcher Guidelines wird gegen die Ärzteschaft als Qualitätskriterium ausgespielt. Der erklärten und versteckten Interessenkonflikte waren und sind viele [12]. Im Rahmenprogramm der erwähnten Veranstaltungen und Kongresse kamen FFF (food, flattery and finance) stets gut an - bis die FMH 1991, die Schweizerische Akademie der Medizinischen Wissenschaften (SAMW) 2006, rev. 2012, und Swissmedic 2006 der Munifizenz der Industrie und dem korrumpierenden Sponsoring der Ärzte einen engeren
Rahmen zu setzen versuchten [9]. Im kleinstaatlichen Rahmen werden die politischen Einflussmöglichkeiten nicht vernachlässigt. So hat sich 2015 in Berner LobbyKreisen neu eine «IG für biomedizinische Forschung und Innovation» konstituiert. Ihr Sekretariat ist bei Interpharma angesiedelt (Generalsekretär Thomas Cueni). Es gehören ihr ein gutes Dutzend bürgerliche Parlamentarier an (www.lobbywatch.ch), welche auch in der schweizerischen Gesundheitskommission vertreten sind.

\section{Big Pharma: neues Geschäftsmodell}

\section{Wie war's bis anhin?}

Das herkömmliche Modell wird in der Online-Version näher vorgestellt. Seine Mängel sind systembedingt, schmälerten aber bisher die Verkäufe dank flächendeckendem, aggressivem Marketing kaum. Dementsprechend übersteigt der Aufwand von internationalen Pharmafirmen für Marketing und Administration den für Forschung und Entwicklung ausgewiesenen bei weitem [1,4].

Das Blockbuster-Modell scheint in absehbarer Frist ausgereizt zu sein. Die firmeneigene und akquirierte Innovationskraft lahmt, die Pipeline für ähnlich konzipierte Mittel droht in absehbarer Zeit auszutrocknen. Einer breiteren Öffentlichkeit wird allmählich bewusst, dass dem Patienten, den sozialen Institutionen und dem Staat während Jahren skandalös viel Geld abgeknöpft worden ist. Eine engmaschigere Regulierung ist die Folge. Die Kriegskassen von Big Pharma sind gut gefüllt und nehmen zusammen mit verwandten Industrien unter der Flagge PM neue Investitionsfelder ins Visier.

\section{Neues Modell: One Patient, One Drug?}

Die Personalisierte Medizin (PM) unternimmt den Versuch, in einem ersten Schritt den individuellen Patienten, die Krankheitsursache und seinen Medikamentenstoffwechsel gentechnologisch präziser zu erfassen. In einem zweiten Schritt sucht sie nach spezifischen Angriffspunkten für eine gezielte medikamentöse Therapie. Es entsteht eine Modellvorstellung, wobei das Hauptinteresse den Biomarkern, d.h. den verantwortlichen Genen (Genotyp), deren Aktivität und den Genprodukten gilt. Näher an das Erscheinungsbild (Phänotyp) heranführen sollen zusätzlich erhobene Daten aus dem persönlichen und weiteren Umfeld, was in der OnlineVersion vertieft diskutiert wird (Big Data, Data Mining). Es resultieren so kleinere Patienten-Zielgruppen für klinische Studien und eine definitive Behandlung (stratifizierte Medizin) [14]. Das Aussortieren von homogenen Kleingruppen enthebt eine Vielzahl von ungeeigneten Patienten (Non-Responders) einer nutzlosen Behandlung, sollte weniger Nebenwirkungen verur- 
sachen, dürfte aber wie gewohnt keine definitive Heilung erreichen. Kommerziell lukrativ und halbwegs aussichtsreich sind Onkologiepatienten, welche mit biotechnologisch hergestellten, überteuerten Mitteln behandelt werden. "Ausserhalb der Onkologie ist die Evidenzbasis für vergleichbare Therapien noch gering. Die PM sollte daher nicht überverkauft werden» (Prof. Vincent Mooser, Leiter der Biomedizin am CHUV in Lausanne, früher bei GlaxoSmithKline; zit. nach [9]). Meiner Meinung nach schiessen die derzeit an die Ärzteschaft gerichteten Forderungen und Prognosen bezüglich PM weit über das absehbare Ziel hinaus. Was an fachlichen Erwartungen (Diagnostikaufwand, Beratungsgesprächen, Qualitätskontrollen, Fortbildung) an sie herangetragen wird, ist eine Zumutung angesichts der Vorwegnahme von verheissungsvollen, aber unbewiesenen Forschungsergebnissen.

Die Bevölkerung scheint sich schon jetzt damit abfinden zu müssen, dass sie immer mehr medikalisiert, stärker finanziell belastet und auf allen Medienkanälen gefügig gemacht werden soll. Es geht schliesslich um Arbeitsplätze.

\section{Personalisierte Medizin: Ausblick}

\section{«Fürsorge ist der Kerngedanke der Medizin.» Nikola Biller-Andorno, 2015 [17]}

Versuchen wir eine vorläufige Bilanz zu ziehen. Ich habe mir vorgenommen, das Projekt der Personalisierten Medizin (PM) zu skizzieren und potente Triebkräfte einzublenden.

So wie sich das Konzept der PM anfangs 2016 präsentiert, wird es einen Forschungsbedarf über das akademische Spektrum hinaus auslösen. Angewandte und Grundlagenforschung sind in gleichem Mass gefragt. Im Herbst vergangenen Jahres hat deshalb die ETH Zürich unter Präsident Lino Guzzella einen Führungsanspruch für Forschung und Entwicklung der personalisierten Medizin in der Schweiz angemeldet. Partner im Projekt sind vorerst die Universitäten Zürich, Basel und della Svizzera Italiana (USI) [18-21]. Physician Scientists erhalten eine Basisausbildung und interessante Startmöglichkeiten. Bedeutend weniger dürfte herausschauen für die Förderung des praktizierenden Ärztenachwuchses. Die Erwartungen auf einen konkreten Patientennutzen der PM bewegen sich zwischen «hope and hype». Die Gesundheitskosten und Versicherungsprämien werden natürlich weiter steigen.

Grosse Vorbehalte sind anzumelden bezüglich der Geschäftspraktiken von Grossfirmen, welche sich an der PM beteiligen, allen voran der pharmazeutischen Industrie. Sie überschreiten seit den 80er Jahren den legalen, wirtschaftlich gerechtfertigten, medizinisch und ethisch verantwortbaren Rahmen. Bisherige Sanktionen wurden mit exorbitanten Gewinnen verrechnet und haben zuwenig gegriffen [22, 23]. An der offerierten Produktpalette, am Zulassungsverfahren der Medikamente, an der Preisgestaltung, am Indikationsbereich der Präparate und am unverhältnismässigen Marketing-Aufwand ist grundsätzliche Kritik anzumelden. Die internationale pharmazeutische Industrie hat das Gesundheitswesen überall, bis in die Akademie hinein, recht eigentlich korrumpiert. Leider ist unser Ärztestand mit ihr symbiotisch verflochten. Wenn der Einfluss von Big Pharma im Projekt PM nicht zurückgebunden wird, wird sich der Schaden am Gemeinwohl vergrössern [1].

\section{Verbesserungspotential ist da. Wo kann man ansetzen?}

Im Zulassungsverfahren für neue Medikamente müssen klinische Studien einer industrieunabhängigen Instanz unterstellt werden [4, 24]. Das neue Mittel ist in erster Linie gegenüber einem bereits eingeführten, billigeren Mittel und nicht bloss gegen Plazebo zu testen. Ein Vergleich mit möglichen nicht-medikamentösen Behandlungen gehört hinein. Neue Mittel sind nicht grundsätzlich nötig oder besser. Negative Studien sind zu publizieren. Wenn sich die Pharmakogenetik durchsetzt und kleinere, homogenere Patientengruppen untersucht werden, muss ein angepasstes Studienverfahren genau untersucht und genehmigt werden.

Wir betreiben zu viel Polypharmazie, schaden damit und wissen zu wenig über die Wechselwirkungen der Medikamente untereinander. Therapeutische Zielsetzungen sind präziser zu deklarieren und zu überprüfen. Kosten-Nutzen-Überlegungen haben einen grossen und in Spezialfällen prohibitiven Stellenwert (Onkologie, Zystische Fibrose).

Die ärztliche Fortbildung muss industrieunabhängig werden. Was Big Pharma leistet, verzerrt die Perspektive, vermittelt bewusste Desinformation und dient in allererster Linie dem Marketing. Die kritischen, umfassend recherchierten Analysen von Marcia Angell [4] und Peter C. Gøtzsche [5] verdienen es, im Lernzielkatalog des Medizinstudiums und in der späteren Weiterbildung Pflichtlektüre zu werden.

Eine dominante Ausrichtung des Gesundheitswesens mit biotechnologisch personalisierter Medizin auf Konkurrenz und Marktökonomie statt auf das Wohlergehen und die Lebensperspektive des Patienten ist qualitativ und wirtschaftlich deletär [17, 25]. Die Patienten und alle, welche sie verantwortungsvoll betreuen, werden das zu entgelten haben. Sie werden schon jetzt mit zunehmendem administrativem Aufwand und scheinheiliger Qualitätskritik belohnt. 
Die redaktionelle Verantwortung für das Literaturverzeichnis liegt beim Autor.

1 Donna Dickenson (Prof. em. of Medical Ethics, University of London), ME Medicine vs. WE Medicine, Reclaiming Biotechnology for the Common Good, Columbia University Press 2013.

2 Urs A. Meyer (Prof. em. Biozentrum Basel), Personalized Medicine: A Personal View, Clinical Pharmacology \& Therapeutics (Perspectives), Vol. 51, No. 5, March 2012, 373-5.

3 Google-Search vom 22.12.15 (www.pmlive.com/top_pharma_list/ global_revenues.

4 Marcia Angell (former Chief Editor of the New Engl J of Medicine), The Truth about Drug Companies, How they deceive us and what to do about it, Random House Trade Paperbacks, New York 2004,2005; deutsch: Der Pharma-Bluff, Wie innovativ die Pillenindustrie wirklich ist, KomPart-Verlag.

5 Peter C. G $\varnothing$ tzsche (Prof. für Forschungsdesign und Forschungsanalyse der Universität Kopenhagen und Leiter des Nordic Cochrane Centers), Deadly Medicines and Organised Crime: How Big Pharma has corrupted healthcare, Radcliffe Publ. Ltd. 2013; dtsch Tödliche Medizin und organisierte Kriminalität - wie die Pharmaindustrie das Gesundheitswesen korrumpiert, Riva-Verlag 2013.

6 Peter J. Meier-Abt (Prof. Dr. med. Uni Basel, Präsident der SAMW), Informationsforum «Zukunft der Medizin - Behandlung nach Mass», Bessere Arzneimittel, gezieltere Arzneimittel, sicherere Arzneimittel, kostengünstigere Therapien, Basel 20. Mai 2010.

7 Antoine de Torrenté und Klaus Neftel, Dr. Knock im 21. Jahrhundert: wie Johnnie Walker «still going strong», «Disease mongering" and Skrabaneks "septicemia», Schweiz. Medizin Forum 2006; 6:1021-22.

8 Christopher Lane, Shyness, How Normal Behavior Became a Sickness, Amer J. of Psychiatry, October 1991.

9 Anne Eckhardt (risicare GmbH), Alexander A. Navarini, Alecs Recher, Klaus Peter Rippe, Bernhard Rütsche, Harry Telser et al., Personalisierte Medizin, 2014 vdf Hochschulverlag AG an der ETH Zürich (Publikation von TA Swiss, Zentrum für Technologiefolgenabschätzung) ISBN 978-3-7281-3591-9.

10 Rainer M. Kaelin, Instrumentalisierung ärztlicher Experten, Schweizerische Ärztezeitung 2015;96(18):663-5.

11 Thomas Cueni (Replik Interpharma), Ein unvollständiges und unausgewogenes Bild, Schweizerische Ärztezeitung 2015;96(18): 666-7.
12 Marcia Angell, Drug Companies \& Doctors, A Story of Corruption, The New York Review of Books, January 19, 2009.

13 Emilio La Rosa, Les vendeurs de maladies, Comment l'industrie pharmaceutique prospère en nous manipulant, Fayard Paris 2011.

14 Alan Niederer, Klinische Studien am einzelnen Patienten, NZZ (Forschung und Technik) vom 20.11.2015

15 Akademien der Wissenschaften der Schweiz (Hrsg.), Big Data im Gesundheitswesen, White Paper, Open Access Publikation 2015, ISSN (online) 1807-2297.

16 Felicitas Witte (Dr. med., medizinische Journalistin), Wir brauchen einen liberalen Umgang mit den Patientendaten, Schweiz Ärztezeitung 2015;96(14-15):537-9. Dieselbe, Big Data: Horrorszenario oder grosse Chance? Schweiz Ärztezeitung 2015; 96(3):75-7.

17 Bruno Kesseli (Interview mit Frau Prof. Nikola Biller-Andorno, Biomedizinische Ethik, Uni Zürich), Fürsorge ist der Kerngedanke der Medizin, Schweiz Ärztezeitung 2015;96(3):75-7.

18 Simon Hehli (Interview mit ETH-Präsident Prof. L. Guzzella), Ohne Medizin kein Spitzenplatz, NZZ vom 12.09.2015.

19 Walter Bernet, Mehr Spitäler für die Ausbildung gewinnen, Uni-Rektor Michael Hengartner begrüsst die neue HochschulKooperation im Bereich des Medizinstudiums, NZZ vom 12.09.2015.

20 Alan Niederer, Nützliche Produkte aus der Forschung, Eröffnungsfeier für das von der ETH und der Universität betriebene Zentrum «Wyss Zurich», NZZ vom 8.12.2015.

21 Hans Stalder (Prof. Honoraire, Faculté de Médecine, Genève), Von der Leiche zum Roboter - warum die ETHs sich täuschen, Schweiz Ärztezeitung 2015;96(46):1708.

22 Nicholas Kristof, When Crime Pays: J+J's Drug Risperdal, New York Times Sept. 17, 2015.

23 Dominik Feldges, Novartis unter dreifachem Verdacht, NZZ 31.3.2016, S. 28 und 36.

24 Michael J. Joyner (M.D., Mayo Clinic Rochester), Seven Questions for Personalized Medicine, JAMA September 8, 2015, 314; 999-1000.

25 Hans Stalder (Prof. Honoraire, Faculté de Médecine, Genève), Beat Bürgenmeier (Prof. em. Geneva School of Economics and Management), Ein freiheitliches Gesundheitswesen, Gastkommentar in der NZZ vom 25.02.2016, S. 11. 